\title{
Uncertainty Risk Assessment of User - side Flexible Resource Scheduling Based on Entropy Weight - TOPSIS Method
}

\author{
Fan Wen ${ }^{1, a}$, Ze Sun ${ }^{2, b}$, Yi Liang ${ }^{2, c^{*}}$ and Dongxiao $\mathrm{Niu}^{2, \mathrm{~d}}$ \\ ${ }^{1}$ Economic and Technological Research Institute, State Grid Zhejiang Electric Power Company \\ LTD., Hangzhou 310008, China \\ ${ }^{2}$ School of Economics and Management, North China Electric Power University, Beijing 102206, \\ China \\ aseefunwen@126.com, ${ }^{\mathrm{b}}$ vision_9366@163.com, ${ }^{\mathrm{c} *}$ lianglouis@126.com, ${ }^{\mathrm{d}}$ niudx@126.com
}

\section{Keywords: Interruptible load management; Uncertainty risk; Entropy weight; TOPSIS}

\begin{abstract}
Interruptible load management utilizes user flexibility to relieve power shortage during peak load, which can avoid or reduce the investment of high power spinning reserve and meet the increase of electricity demand. There are lots of uncertain risk factors in the process of interruptible load management, so we must consider the uncertain risk of user side flexible resource scheduling. This article sets up the uncertainty risk evaluation index system for flexible scheduling of users on the basis of various uncertainties in technical, economic and social risks, and then illustrates the principle and steps of entropy-TOPSIS model. Finally, we use the model for empirical analysis and calculation, and draw the corresponding conclusions.
\end{abstract}

\section{Introduction}

The particularity of electric energy determines that the power grid must be planned in advance to ensure the reliability of the system operation. With the expansion of power system scale, the continuous growth of power demand and the deepening and development of power market reform, the power system is approaching its limit. The traditional day-ahead scheduling can only achieve power balance by optimizing the dispatching of generation-side resources. This model can no longer meet the current energy shortage and power shortage situation.

Interruptible load management is based on the basic idea of fully mobilizing the electricity elasticity of the user to eliminate or alleviate the tense situation of electric peak power supply. It uses user's flexibility to relieve power shortage during peak load, so as to avoid or reduce the investment of high power spinning reserve and meet the increase of power demand. From the interruptible load management market operation process, we can see that the power companies must weigh the advantages and disadvantages to evaluate the users comprehensively before subscribing with the users, so as to select the declared users who can maximize the interests of power companies. However, there are many uncertainties in the decision of electric power companies to sign interruptible load contracts with users, which not only directly affects the economic benefits of power companies, but also causes risk losses. Due to many uncertainties, it is almost impossible to accurately measure the total cost and total revenue generated by each reporting user over the entire contract period at this stage. At the same time, different declaring users may also have various uncertain risk factors. Therefore, we need to evaluate the three factors of user side flexible resource scheduling, that is, the technical, economic and social risks of interruptible load management.

TOPSIS is mainly used in multi-objective decision-making and risk assessment research[1]. The evaluation effect of this method is not affected by the evaluation index and the number of evaluation objects. This method has the most obvious advantages in the evaluation of a project with a large number of indicators but with a small evaluation scale. In addition, it can perform vertical and horizontal comparative analysis of each evaluation object and has the advantages of simple calculation. Entropy weights are weighted according to the original data and are objective and truthful[2]. In view of this, this paper selects three factors of uncertainty, including technology, economy and social risk, to build 
evaluation index system, then preprocessing indicators and determining index weights. Finally, we use the TOPSIS method to evaluate the uncertainty risk and draw the conclusion.

\section{Construction of Uncertainty Risk Evaluation Index System for User Flexible Resource Scheduling}

The uncertainty risk assessment of user side flexible resource scheduling is a complex process. It involves many aspects. We should consider all kinds of uncertain factors and evaluate all kinds of risks, so as to make the best decision. Based on the relevant literature and the analysis of the status quo, this paper selects and establishes the index through the uncertainty of technical, economic and social risks.

Uncertainty Technical Risk. The risk of uncertain technical risk is mainly considering the difficulty of technical implementation in the management of interruptible load. The interruptible load management in our country is in its infancy, and many supporting facilities and related process mechanisms are immature. Therefore, the implementation of electric power companies must consider all kinds of risks existing in technology. The main consideration of the technology implementation risks are hardware defects, software defects and process mechanism defects. The main consideration of hardware defects is the failure rate and wear rate of hardware devices. Software defects consider the maturity of optimization modeling techniques. The defects of process mechanism are the reasonableness of market operation process and the promptness of interrupt signal transmission.

Uncertainty Economic Risk. Uncertainty Economic factors mainly relate to the costs and benefits of implementing interruptible load management. Cost factors include disbursement of compensation to users, investment costs of related equipment, operating costs of related equipment and implementation of electricity sales losses. Revenue factors include avoidable cost of electricity generation in the electricity market, avoidable cost of investment in power transmission and distribution equipment and avoidable cost of operating transmission and distribution equipment.

Uncertainty Social Risk. The uncertainty of social factors mainly involves policy changes and users' default risk. User default risk is concerned with user's production and operation status, user integrity and the binding force of the interruptible load management contract to the user's breach of contract. The risk of policy change can be divided into the risk of the change of the power policy and the risk of the change of the industry policy of the user.

Construction of Index System. According to the above analysis, the index system and index types of risk assessment of flexible resource scheduling constructed on the user side are shown in Table 1.

Table 1 Uncertainty risk evaluation index system for flexible resource scheduling on user side

\begin{tabular}{|c|c|c|}
\hline Primary indicator & Secondary indicators & Indicator type \\
\hline \multirow{5}{*}{ Technical risk $\mathrm{C}_{1}$} & Hardware equipment failure rate $C_{11}$ & Minimum \\
\hline & Hardware wear rate $\mathrm{C}_{12}$ & Minimum \\
\hline & Optimization Modeling Technology Proficiency $\mathrm{C}_{13}$ & Maximum \\
\hline & Rationality of market operation process $\mathrm{C}_{14}$ & Maximum \\
\hline & Interrupt signal to convey timeliness $\mathrm{C}_{15}$ & Maximum \\
\hline \multirow{7}{*}{ Economic risk $\mathrm{C}_{2}$} & Interrupt compensation costs $\mathrm{C}_{21}$ & Minimum \\
\hline & Equipment investment costs $\mathrm{C}_{22}$ & Minimum \\
\hline & Equipment operating costs $C_{23}$ & Minimum \\
\hline & User electricity sale loss $\mathrm{C}_{24}$ & Minimum \\
\hline & Avoidable cost of power generation market purchase $C_{25}$ & Maximum \\
\hline & $\begin{array}{l}\text { Avoidable investment cost of power transmission and distribution } \\
\text { equipment } \mathrm{C}_{26}\end{array}$ & Maximum \\
\hline & $\begin{array}{l}\text { Avoidable operation cost of power transmission and distribution } \\
\text { equipment } \mathrm{C}_{27}\end{array}$ & Maximum \\
\hline \multirow{5}{*}{ Social risk $\mathrm{C}_{3}$} & Status of user production and operation $\mathrm{C}_{31}$ & Maximum \\
\hline & Trustworthiness of the user $\mathrm{C}_{32}$ & Maximum \\
\hline & The binding force of the contract to the user's breach of contract $\mathrm{C}_{33}$ & Maximum \\
\hline & Risk of power policy changes $\mathrm{C}_{34}$ & Minimum \\
\hline & The risk of policy changes in the industry of the user $\mathrm{C}_{35}$ & Minimum \\
\hline
\end{tabular}




\section{Entropy Weight - TOPSIS Comprehensive Evaluation Model}

Entropy Weight Method. It is known from the concept of entropy that the decision accuracy can be improved by relying on the decision information quantity. Entropy is a very ideal scale in multi-objective decision and evaluation [3].For a given evaluation index, the greater the difference between the different sample data, the greater the impact of the index on the evaluation results. That is, the more information contained and transmitted by the index, the greater the weight it will be given [4].Therefore, according to the degree of variation of each index, the weight of each evaluation index can be calculated with the information entropy.

Establish a multi-objective decision matrix

$$
A=\left(\begin{array}{ccc}
x_{11} & \ldots & x_{1 n} \\
\vdots & \ddots & \vdots \\
x_{m 1} & \cdots & x_{m n}
\end{array}\right)
$$

In $A=\left(x_{i j}\right)(i=1,2, \ldots, m, j=1,2, \ldots, n), \mathrm{m}$ and $\mathrm{n}$ represent the level of indicators and the number of indicators, respectively. The $x$ represents the importance of the $\mathrm{j}$ component of the $\mathrm{i}$ index level.

Normalized score

$$
P_{i j}=x_{i j} / \sum_{i=1}^{m} x_{i j}
$$

Calculate the entropy of the jth indicator

$$
e_{j}=-\frac{1}{\ln m} \sum_{i=1}^{m}\left(P_{i j} \cdot \ln P_{i j}\right) \quad(j=1,2, \ldots, n)
$$

Calculate the difference coefficient of the jth index

$$
g_{j}=1-e_{j}
$$

Determine the weight of the index

$$
w_{j}=\frac{g_{j}}{\sum_{1}^{n} g_{j}}
$$

TOPSIS Method. TOPSIS means "approximation ideal solution sort method", which is a commonly used decision-making technique in system engineering. It is mainly used to solve the multi-objective decision-making problem of finite solutions. It is a comprehensive evaluation method using distance as the evaluation criterion [5]. By defining a measure in the target space, we calculate the degree of the target's close/deviating from the positive and negative ideal solution, which can evaluate the uncertainty risk degree of the flexible resource scheduling on the user side, and provide corresponding suggestions for decision makers in order to make better decisions.

Entropy Weight - TOPSIS Model. After giving weight of each evaluation index by entropy weight method, we can use TOPSIS method to carry out comprehensive evaluation on the uncertain risk of user side flexible resource scheduling, and the corresponding evaluation procedures are as follows.

Weighted calculation of initial data

$$
R=\left(\begin{array}{ccc}
P_{11} w_{1} & \ldots & P_{1 n} w_{n} \\
\vdots & \ddots & \vdots \\
P_{m 1} w_{1} & \cdots & P_{m n} w_{n}
\end{array}\right)=\left(\begin{array}{ccc}
r_{11} & \ldots & r_{1 n} \\
\vdots & \ddots & \vdots \\
r_{m 1} & \cdots & r_{m n}
\end{array}\right)
$$

The $m$ represents the level of evaluation index, and the $n$ represents the number of evaluation index.

Calculate positive ideal solution and negative ideal solution 
The positive solution is the hypothetical value of the optimal value in the ideal case, and the negative ideal solution is the opposite, that is, the virtual solution of the worst value, and its calculation method is as follows:

$$
\begin{aligned}
& r_{j}^{+}=\left\{\left(\max _{1 \leq j \leq m} r_{i j} \mid j \in J_{1}\right),\left(\min _{1 \leq j \leq m} r_{i j} \mid j \in J_{2}\right) \mid i=1,2, \ldots, m\right\} \\
& r_{j}^{-}=\left\{\left(\min _{1 \leq j \leq m} r_{i j} \mid j \in J_{1}\right),\left(\max _{1 \leq j \leq m} r_{i j} \mid j \in J_{2}\right) \mid i=1,2, \ldots, m\right\}
\end{aligned}
$$

The benefit type index set is represented by the $J_{1}$, and the cost type set is represented by the $J_{2}$. The positive and negative ideal solutions are represented by $r_{j}^{+}$and $r_{j}^{-}$, respectively.

Then, the Euclidean distance is obtained as follows:

$$
\begin{aligned}
& d_{1}^{+}=\sqrt{\sum_{j=1}^{n}\left(r_{i j}-r_{j}^{+}\right)^{2}}(i=1,2, \ldots, m) \\
& d_{1}^{-}=\sqrt{\sum_{j=1}^{n}\left(r_{i j}-r_{j}^{-}\right)^{2}}(i=1,2, \ldots, m)
\end{aligned}
$$

Determination of relative proximity

$$
C_{i}=\frac{d_{i}^{+}}{d_{i}^{+}+d_{i}^{-}}(i=1,2, \ldots, m)
$$

The smaller the distance we get, the more the evaluation object will be closer to the ideal level, which indicates that the object of evaluation object has more superiority and the ranking naturally goes forward.

\section{Empirical Research}

This paper selects the corresponding data in the interruptible load management of State Grid Zhejiang Electric Power Corporation, and invites 20 experts in the field to score indicators, and then gets the membership degree of each index. The evaluation level is divided into four grades: excellent, good, medium and bad. We draw lessons from other provinces' uncertain risk assessment standard of interruptible load management to determine scoring criteria and score. The method of determining the membership degree of the index is as follows:

$$
x_{i}=\frac{1}{n} \sum_{m=1}^{n} u_{m}
$$

In the above calculation method: the comprehensive score of the $\mathrm{i}$ index is represented by $x_{i}$; the $u_{m}$ is the actual score of the $\mathrm{m}$ expert on a selected index; the $\mathrm{n}$ represents the number of experts.

The results obtained by the above steps are shown in Table 2. 
Table 2 Evaluation index score sheet

\begin{tabular}{|c|c|c|c|c|c|}
\hline \multicolumn{2}{|c|}{ Evaluation index } & Excellent & Good & Medium & Bad \\
\hline \multirow{4}{*}{$\mathrm{C}_{1}$} & $\mathrm{C}_{11}$ & 0.55 & 0.30 & 0.10 & 0.05 \\
\cline { 2 - 6 } & $\mathrm{C}_{12}$ & 0.35 & 0.40 & 0.20 & 0.05 \\
\cline { 2 - 6 } & $\mathrm{C}_{13}$ & 0.40 & 0.40 & 0.15 & 0.05 \\
\cline { 2 - 6 } & $\mathrm{C}_{14}$ & 0.45 & 0.35 & 0.10 & 0.10 \\
\cline { 2 - 6 } & $\mathrm{C}_{15}$ & 0.45 & 0.30 & 0.15 & 0.10 \\
\cline { 2 - 6 } & $\mathrm{C}_{21}$ & 0.40 & 0.35 & 0.10 & 0.15 \\
\cline { 2 - 6 } $\mathrm{C}_{2}$ & $\mathrm{C}_{22}$ & 0.50 & 0.25 & 0.20 & 0.05 \\
\cline { 2 - 6 } & $\mathrm{C}_{23}$ & 0.45 & 0.30 & 0.10 & 0.15 \\
\cline { 2 - 6 } & $\mathrm{C}_{24}$ & 0.40 & 0.40 & 0.10 & 0.10 \\
\cline { 2 - 6 } & $\mathrm{C}_{25}$ & 0.55 & 0.35 & 0.05 & 0.05 \\
\cline { 2 - 6 } & $\mathrm{C}_{26}$ & 0.40 & 0.35 & 0.20 & 0.05 \\
\hline $\mathrm{C}_{3}$ & $\mathrm{C}_{27}$ & 0.35 & 0.50 & 0.10 & 0.05 \\
\cline { 2 - 6 } & $\mathrm{C}_{31}$ & 0.40 & 0.40 & 0.10 & 0.10 \\
\cline { 2 - 6 } & $\mathrm{C}_{32}$ & 0.20 & 0.30 & 0.40 & 0.10 \\
\cline { 2 - 6 } & $\mathrm{C}_{33}$ & 0.30 & 0.45 & 0.10 & 0.15 \\
\cline { 2 - 6 } & $\mathrm{C}_{34}$ & 0.50 & 0.25 & 0.10 & 0.15 \\
\hline
\end{tabular}

According to Eq.3, Eq.4 and Eq.5, we calculate the entropy and difference coefficient of each index, and finally get the final weight of each index. The results are shown in Table 3.

Table 3 Evaluation index weight, positive and negative ideal solution

\begin{tabular}{|c|c|c|c|c|}
\hline \multicolumn{2}{|c|}{ Evaluation index } & Index weight & Positive ideal solution & Negative ideal solution \\
\hline \multirow{4}{*}{$\mathrm{C}_{1}$} & $\mathrm{C}_{11}$ & 0.0592 & 0.0326 & 0.0030 \\
\cline { 2 - 5 } & $\mathrm{C}_{12}$ & 0.0576 & 0.0230 & 0.0029 \\
\cline { 2 - 5 } & $\mathrm{C}_{13}$ & 0.0580 & 0.0232 & 0.0029 \\
\cline { 2 - 5 } & $\mathrm{C}_{14}$ & 0.0604 & 0.0272 & 0.0060 \\
\cline { 2 - 5 } & $\mathrm{C}_{15}$ & 0.0588 & 0.0265 & 0.0059 \\
\cline { 2 - 5 } & $\mathrm{C}_{21}$ & 0.0585 & 0.0234 & 0.0059 \\
\cline { 2 - 5 } & $\mathrm{C}_{22}$ & 0.0580 & 0.0290 & 0.0029 \\
\cline { 2 - 5 } $\mathrm{C}_{2}$ & $\mathrm{C}_{23}$ & 0.0588 & 0.0265 & 0.0059 \\
\cline { 2 - 5 } & $\mathrm{C}_{24}$ & 0.0603 & 0.0241 & 0.0060 \\
\cline { 2 - 5 } & $\mathrm{C}_{25}$ & 0.0605 & 0.0333 & 0.0030 \\
\cline { 2 - 5 } & $\mathrm{C}_{26}$ & 0.0576 & 0.0230 & 0.0029 \\
\hline \multirow{5}{*}{$\mathrm{C}_{3}$} & $\mathrm{C}_{27}$ & 0.0589 & 0.0295 & 0.0060 \\
\cline { 2 - 5 } & $\mathrm{C}_{31}$ & 0.0603 & 0.0241 & 0.0058 \\
\cline { 2 - 5 } & $\mathrm{C}_{32}$ & 0.0578 & 0.0231 & 0.0059 \\
\cline { 2 - 5 } & $\mathrm{C}_{33}$ & 0.0588 & 0.0265 & 0.0029 \\
\cline { 2 - 5 } & $\mathrm{C}_{34}$ & 0.0580 & 0.0290 & 0.0234 \\
\hline
\end{tabular}

From the previous article, the evaluation grade of the index is divided into four as follows: excellent, good, medium and poor, and there are 17 evaluation indexes. According to the Eq.6, we get the weighted set of weights. Then, according to the Eq.7 and the Eq.8, we can calculate the positive and negative ideal solutions of each index, and the results are shown in Table 3.

According to the Eq.9 and the Eq.10, the Euclidean space distance of four index grades of excellent, good, medium and bad is carried out respectively. Then according to the Eq.11, the relative proximity between the index grade and the positive ideal solution and the negative ideal solution is calculated. The relative proximity of the calculation is detailed in Table 4.

Table 4 Relative degree of index grade

\begin{tabular}{|c|c|}
\hline Indicator level & Relative proximity \\
\hline Excellent & 0.1678 \\
\hline Good & 0.3177 \\
\hline Medium & 0.7529 \\
\hline Bad & 0.9383 \\
\hline
\end{tabular}


Because the object of evaluation and ranking the relative proximity index grade have the following relationship: numerical relative approach degree is bad for the extent of the object of evaluation ranking, on the other hand, the numerical relative approach degree is smaller to the extent of the object of evaluation ranking. Therefore, based on the above results, we can draw a conclusion that the comprehensive risk assessment results of Zhejiang's interruptible load management are excellent, that is, user side flexible resource scheduling has small uncertainty and low risk.

\section{Conclusion}

In this paper, we consider various uncertainties in terms of technical, economic and social aspects of implementing interruptible load, and establish an uncertainty risk assessment index system for flexible resource scheduling on the user side. By constructing entropy weight-TOPSIS model, the level of uncertainty risk of flexible resource scheduling on user side is evaluated. Entropy weight method is used to determine the weight. Compared with those subjective weight methods, entropy weight method has higher weighting accuracy and objectivity. Combined with TOPSIS method for risk assessment, it can objectively and truly reflect the degree of risk. In the end, we take the State Grid Zhejiang Power Company as an example to evaluate the uncertainty risk of interruptible load management. The result is excellent, that is, the risk of flexible scheduling of resources on the user side is low.

\section{Acknowledgements}

This work is supported by the technology project of Economic and Technological Research Institute, State Grid Zhejiang Electric Power Company LTD (Project name: "Research on Flexible and Intelligent Energy Supply and Consumption Ecological Community in Electricity Market”).

\section{References}

[1] C. Wang, F.C. Jiang and Q.D. Ma: Journal of Safety and Environment, Vol. 16 (2016) No.3, p.33.( In Chinese)

[2] X.P. Lei and G.H. Qiu: Acta Scientiae Circumstantiae, Vol. 36 (2016) No.1, p.314.( In Chinese)

[3] X.P. Lei, Y. Wu, S. YE, Z.R. Zhao and L. Wang: Transactions of the Chinese Society of Agricultural Engineering, Vol. 28 (2012) No.2, p.233.( In Chinese).

[4] Y.H. QIU: Management Decision Entropy and Its Application (China Electric Power Press, China 2010) (In Chinese)

[5] Y. Zheng and Y. Chen: Journal of Chongqing University of Technology (Natural Science), Vol. 29 (2015) No.1, p.85.( In Chinese) 